\title{
Intervencionismo en los sectores exportadores tradicionales durante el Primer Franquismo: el caso del corcho
}

\author{
Interventionism in the traditional export sectors during the first \\ Franco period: the case of cork
}

\author{
FRANCISCO MANUEL PAREJO MORUNO' \\ Universidad de Extremadura \\ fmparejo@unex.es
}

\begin{abstract}
Resumen: Este trabajo pone de manifiesto los efectos negativos del intervencionismo cambiario sobre los sectores exportadores abordando el estudio de la industria corchera española durante la autarquía franquista. Se analiza cómo el mantenimiento de un tipo de cambio oficial sobrevaluado generó problemas competitivos para esta industria, llevándole a perder el liderazgo en el mercado mundial. Asimismo, se estudia el programa selectivo de subsidios directos a la exportación que implementó la dictadura franquista para contrarrestar los negativos efectos de la intervención cambiaria sobre la exportación, el cual generó numerosas ineficiencias en este sector exportador tradicional.
\end{abstract}

Palabras clave: Corcho, industria corchera, dumping, tipo de cambio, subsidios a la exportación.

\begin{abstract}
This paper attempts to highlight the negative effects of the currency intervention on export sectors. To this aim, it is proposed as a case study the Spanish cork industry during the Franco autarchy. It is discussed how to maintain an overvalued official exchange generated competitive problems to the Spanish cork industry, leading to lose the leadership in the global market. Furthermore, it is studied a selective program of direct export subsidies that implemented the Franco dictatorship to counteract the negative effects of foreign exchange intervention on export, showing how it generated numerous inefficiencies in this sector.
\end{abstract}

Keywords: Cork, cork industry, dumping, exchange rates, export subsidies.

\footnotetext{
${ }^{1}$ El autor agradece la financiación proporcionada por la Junta de Extremadura a través del Programa de ayudas para la realización de actividades de investigación y desarrollo tecnológico, de divulgación y de transferencia de conocimiento por los Grupos de Investigación de Extremadura (GR15182).
}

Recibido: 4 de diciembre de 2016; aceptado: 24 de marzo de 2017; publicado: 21 de septiembre de 2017. Revista Historia Autónoma, 11 (2017), pp. 219-239.

e-ISSN: 2254-8726; DOI: https://doi.org/10.15366/rha2017.11.011 
Introducción

Es conocida la delicada situación en materia de divisas que afrontaron los primeros gobiernos de la dictadura franquista en España, la cual restringió las posibilidades de importar maquinaria, tecnología y materias primas necesarias para la fabricación ${ }^{2}$. Ello habría retrasado la recuperación de la industria española, frustrando el desarrollo de algunos sectores, como el corchero, en el mercado internacional ${ }^{3}$. El rechazo del régimen franquista a la ayuda americana, primero, el aislamiento diplomático internacional de España decretado por Naciones Unidas en 1945, después, y en general la legislación franquista sobre inversión extranjera hasta 1959 hicieron que la entrada de capital exterior fuese insuficiente, por lo que el fomento de la exportación se convirtió en uno de los caballos de batalla de la dictadura, al ser esta la única vía para allegar los dólares que el país necesitaba para poder importar.

A pesar de que esta reflexión impregna la bibliografía existente al respecto ${ }^{4}$, son poco conocidas las medidas adoptadas por la dictadura para el fomento exportador. Al menos son pocos los trabajos que han concretado tales medidas, o que han valorado sus efectos sobre las exportaciones españolas en su conjunto, o de un sector en particular. Este trabajo pretende avanzar en este conocimiento, realizando un análisis de los instrumentos que la dictadura franquista utilizó para el fomento de las exportaciones corcheras entre julio de 1939 - fecha de la primera intervención al respecto- y 1959, año en que el Plan de Estabilización de la economía española abrió un período de mayor liberalización de los intercambios con el exterior.

El hecho de que el estudio aborde exclusivamente las medidas adoptadas sobre las exportaciones de la rama corchera obliga a contemplar la investigación como un estudio de caso. De esta forma, no se pretende extrapolar los resultados de la investigación al conjunto de los sectores exportadores españoles, en los que la dictadura pudo haber aplicado iguales o diferentes medidas a las que se comentarán. Dicho esto, el negocio corchero constituye una buena elección para valorar el éxito o el fracaso de las medidas del fomento exportador; primero,

\footnotetext{
${ }^{2}$ Catalán, Jordi, "Reconstrucción política económica y desarrollo industrial. Tres economías del sur de Europa, 1944-1953", en Prados de la Escosura, Leandro y Vera Zamagni (eds.), El desarrollo económico en la Europa del Sur. España e Italia en perspectiva histórica, Madrid, Alianza, 1992, pp. 359-395; Martínez Ruiz, Elena, El control de cambios en la España franquista. El Instituto Español de Moneda Extranjera, 1939-1973, tesis doctoral, Universidad de Alcalá de Henares, 2000; Martínez Ruiz, Elena, "Sector exterior y crecimiento en la autarquía", en Revista de Historia Económica, número extraordinario (2001), pp. 229-251; Martínez Ruiz, Elena, "La distribución de las divisas en el sector industrial. Poder público y poder privado en lucha por las divisas", en Historia y Política. Ideas, procesos y movimientos sociales, 9 (2003), pp. 95-122.

${ }^{3}$ Zapata, Santiago, "Del suro a la cortiça. El ascenso de Portugal a la primera potencia corchera del mundo", en Revista de Historia Industrial, 22 (2002), pp. 109-137; Parejo Moruno, Francisco Manuel, "Cambios en el negocio mundial corchero. Un análisis a largo plazo de las exportaciones españolas (1849-2000)", en Historia Agraria, 39 (2006), pp. 241-265.

${ }^{4}$ Véase, por ejemplo, Clavera, Joan et al., Capitalismo español. De la autarquía a la estabilización (1939-1959), Madrid, Edicusa, 1973; Viñas, Ángel et al., Política comercial exterior en España (1931-1975), Madrid, Banco Exterior de España, 1979; Martínez Ruiz, Elena, El control de cambios... op. cit.; Martínez Ruiz, Elena, "La distribución..."op. cit., pp. 95-122; Martínez Ruiz, Elena, "El sector exterior durante la Autarquía. Una reconstrucción de las balanzas de pagos de España (1940-1958)" en Estudios de Historia Económica, 43 (2003), pp. 7-196.
} 
porque fue uno de los sectores en que tales medidas fueron más explícitas; y segundo, porque fue un negocio eminentemente exportador hasta las últimas décadas del siglo $\mathrm{xx}^{5}$, que aún hoy sigue enviando al exterior la mayor parte de su producción ${ }^{6}$. De este carácter exportador da fe la participación del capítulo corchero en las exportaciones españolas, que se mantuvo de forma constante por encima del 2 por 100 en la segunda mitad del siglo XIX, aumentando en el primer cuarto del siglo xx hasta situarse por encima del 7 por 100 a finales de la década de 1920. Hasta esas fechas, el corcho siempre había ocupado uno de los primeros lugares en la exportación de productos agrarios ${ }^{7}$, particularmente en la relativa a los productos forestales ${ }^{8}$.

En efecto, ante la ausencia de una demanda interior significativa, el negocio corchero español fue capaz de protagonizar una espectacular inserción en el mercado internacional desde comienzos del siglo XIX, especialmente a partir de la segunda mitad de dicha centuria, mejorando sus registros exportadores década a década, particularmente en los capítulos de mayor valor añadido9. De esta forma, en el primer tercio del siglo xx la industria corchera española vivió los mejores momentos de su historia en lo que a registros exportadores se refiere, situándose entre las primeras ramas exportadoras del país a finales del decenio de 1920. Dado el declive posterior, acontecido durante las décadas de 1930, 1940 y $1950^{10}$, y la pérdida de peso del corcho en el comercio de exportación a lo largo de ellas, el propósito de este trabajo es analizar las medidas aplicadas por la dictadura franquista para el fomento de las exportaciones corcheras, valorando sus efectos sobre la evolución de aquellas durante el período 1939-1959.

También nos cuestionamos cuáles fueron los objetivos reales de la dictadura al poner en funcionamiento las medidas del fomento exportador. Estos fueron definidos desde las instancias oficiales como la defensa de las industrias exportadoras, entre ellas la del corcho, las cuales acababan de vivir una década para olvidar — la de 1930-, como consecuencia de la crisis iniciada en 1929 y la posterior guerra civil. Sin embargo, como se verá, el resultado final no fue

\footnotetext{
${ }^{5}$ El consumo nacional de manufacturas corcheras no iba más allá del 10 por 100 de las exportaciones hacia 1930; Zapata, Santiago, "El alcornoque y el corcho en España, 1850-1935”, en Garrabou, Ramón et al. (eds.), Historia agraria de la España contemporánea. Vol. 3: El fin de la agricultura tradicional (1900-1960), Barcelona, Crítica, 1986, p. 241. Todavía hacia 1950, poco más del 20 por 100 de la producción de la industria corchera española iba a parar al mercado interior; Parejo Moruno, Francisco Manuel, El negocio de exportación corchera en España y Portugal durante el siglo XX. Cambios e intervención pública, Cáceres, Servicio de Publicaciones de la Universidad de Extremadura, 2009.

${ }^{6}$ Zapata, Santiago, "Del suro a la cortiça..." op. cit.; Sala, Pere, Manufacturas de Corcho S. A. (antiga Miquel \& Vincke). Líder de l'exportació industrial espanyola (1900-1930), Palafrugell, Museu del Suro, 2003; Parejo Moruno, Francisco Manuel, "Cambios en el negocio..." op. cit.; Parejo Moruno, Francisco Manuel, El negocio de exportación... op. cit.; y, especialmente, Zapata, Santiago et al., "Manufacture and trade of cork products. an international perspective", en Aronson, James et al. (eds.), Cork oak woodlands on the edge. Ecology, adaptive management and Restoration, Washington, Island Press, 2009, pp. 189-200.

${ }^{7}$ Gallego, Domingo y Vicente Pinilla, "Del librecambio matizado al proteccionismo selectivo. El comercio exterior de productos agrarios y alimentos en España entre 1849 y 1935 (Segunda parte. Apéndice)”, en Revista de Historia Económica, 3 (1996), pp. 630-639.

${ }^{8}$ Iriarte, Iñaki, "La inserción internacional del sector forestal español, 1849-1935", en Revista de Historia Industrial, 27 (2005), p. 30.

${ }^{9}$ Parejo Moruno, Francisco Manuel, "Cambios en el negocio..." op. cit.

${ }^{10}$ Para apreciar y valorar adecuadamente este declive se recomienda la lectura de Parejo Moruno, Francisco Manuel, "El comercio de exportación corchera en Portugal y España. Una historia secular de éxitos y fracasos", en Zapata, Santiago (ed.), Alcornocales e industria corchera. Hoy, ayer y mañana, Palafrugell, Museo del Suro de Palafrugell, 2009, pp. 782-807.
} 
tanto la protección de la industria ni el relanzamiento de la exportación de manufacturas como la discrecionalidad de las medidas, muchas veces condicionadas por la presión de grupos de interés o por la urgencia de conseguir divisas para paliar la dramática escasez de estas.

Para valorar el comentario anterior, el lector debe conocer una peculiaridad que ha acompañado históricamente a la fabricación del corcho en España. Nos referimos a la dualidad geográfica que ha dividido históricamente los intereses de los agentes corcheros españoles en dos frentes antagónicos ${ }^{11}$ : uno en el suroeste, en las regiones productoras de corcho -Extremadura y Andalucía, principalmente-, que es también donde se ha asentado tradicionalmente la industria preparadora (primera transformación del corcho); y otro en Cataluña, donde se ha ubicado históricamente la industria transformadora del corcho, surgida en el primer tercio del siglo XVIII, y que gozó de una posición hegemónica en el mercado mundial de manufacturas corcheras hasta la guerra civil española, cuando cedió su posición de privilegio a la industria portuguesa $^{12}$.

El primero de estos frentes - los propietarios forestales y los industriales preparadoresha intentado tradicionalmente vender su producción - el corcho sin manufacturar- donde fuera mejor remunerada. Esto solía ocurrir en el mercado internacional, por lo que fue frecuente el recurso a la exportación, perjudicándose la industria nacional, especialmente durante la autarquía franquista, en que fue difícil transportar el corcho desde el suroeste hasta las fábricas catalanas $^{13}$. Ante esta situación, los industriales catalanes siempre habían reclamado a la administración medidas arancelarias frente a la salida al exterior del corcho español, las cuales fueron aplicadas de forma discontinua entre 1787 —año en que se sancionó por primera vez el Arancel de Aduanas en España- y el Arancel Cambó de 1922, en lo que se ha conocido como la "cuestión arancelaria del corcho"14. Conviene retener este enfrentamiento de intereses, pues es clave para entender si las medidas del fomento exportador aplicadas por la dictadura fueron en el sentido de proteger a la industria nacional del corcho, o si por el contrario se vieron neutralizadas por la capacidad de influencia de los lobbies forestales y preparadores en las decisiones públicas en pos a una mayor liberalización de las exportaciones de corcho sin transformar.

Para alcanzar los objetivos descritos, el trabajo se divide en cinco apartados. El primero de ellos es la presente introducción. En el segundo se presenta la evolución de las exportaciones corcheras españolas entre 1929 y 1959, tomándose como patrón de comparación el conjunto de las exportaciones españolas. También se exponen las primeras voces de los corcheros solicitando a la administración un marco protector, y la respuesta dada por esta a la industria.

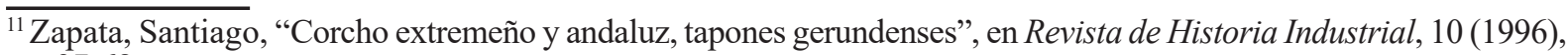
pp. 37-68.

${ }_{12}^{2}$ Zapata, Santiago, "Del suro a la cortiça..." op. cit.

${ }^{13}$ Parejo Moruno, Francisco Manuel, El negocio de exportación... op. cit., pp. 218-231.

${ }^{14}$ Medir, Ramiro, Historia del gremio corchero, Madrid, Alhambra, 1953; Sala, Pere, "Obrador, indústria i aranzels al districte surer català (1830-1930)", en Recerques, 37 (1998), pp. 109-136.
} 
En el tercero se aborda el régimen de subsidios directos a la exportación de productos corcheros que estuvo vigente en España entre 1946 y 1950, y que ocasionó graves problemas diplomáticos y comerciales. En el cuarto se analiza el tratamiento otorgado al corcho en el mecanismo de tipos de cambios múltiples que la dictadura aplicó entre noviembre de 1948 y abril de 1957. Finalmente, el quinto apartado recoge las principales conclusiones de la investigación.

\section{Las primeras medidas de apoyo a la exportación}

En el gráfico 1 se presenta la evolución de las exportaciones españolas en su conjunto y las de la rama corchera en particular entre 1929 y $1959^{15}$. Las primeras experimentaron una intensa y continuada caída entre 1929 y 1942 fruto de la crisis de los años treinta y de los efectos de las guerras civil y mundial. Después de 1942 se recuperaron vigorosamente hasta 1951, año en que entraron en una senda de estancamiento que todavía permanecía al final del período analizado, sin que se recuperasen los niveles de exportación previos a 1929. Con todo, el comportamiento de las exportaciones corcheras fue bastante peor. Su caída fue mucho más vertical e intensa que la de las exportaciones totales. También fue más prolongada, pues no tocaron suelo hasta 1945, momento en que iniciaron una senda creciente que, con altibajos, se mantuvo hasta 1959. Para entonces el valor del corcho enviado al exterior por España no alcanzaba el 50 por 100 del valor exportado en 1929.

Las razones del declive exportador en España son conocidas. Además de los aspectos coyunturales - la gran depresión de los años treinta y las guerras civil y mundial一, confluyeron tres circunstancias desde 1939 que afectaron negativamente a los sectores exportadores. La primera de ellas es la orientación autárquica de la dictadura franquista, que resultó perjudicial para los sectores dependientes del mercado internacional ${ }^{16}$. La segunda es el mantenimiento artificial de una peseta fuerte, esto es, la fijación de un tipo de cambio oficial tremendamente sobrevaluado de la peseta con respecto al dólar USA. Este tipo de cambio, fijado en 1941 en 10,95 pesetas/dólar USA, permaneció invariable hasta 1957, ajeno a la evolución de la cotización de la peseta en el mercado internacional de divisas ${ }^{17}$. En tercer lugar, hay que considerar las

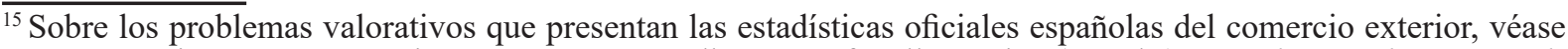
Tena, Antonio, "Sector exterior", en Carreras, Albert y Tafunell, Xavier (coords.), Estadísticas históricas de España, siglos XIX-XX, vol. II, Pamplona, Fundación BBVA, 2005, pp. 590-593. En lo que respecta a las estadísticas comerciales del capítulo corchero, véase Parejo Moruno, Francisco Manuel, El negocio de exportación... op. cit., pp. 34-42.

${ }_{16}^{16}$ Viñas, Ángel et al., Política comercial exterior... op. cit.

${ }^{17}$ Esta afirmación es susceptible de ser matizada, pues es cierto que a finales de las décadas de 1940 y comienzos de 1950 tuvo lugar una cierta devaluación encubierta que mejoró significativamente la competitividad de las exportaciones españolas; Serrano, José María y María Jesús Asensio, "El ingenierismo cambiario. La peseta en los años del cambio múltiple, 1948-1959”, en Revista de Historia Económica, 3 (1997), pp. 545-573.
} 
consecuencias competitivas derivadas de la imposibilidad de importar maquinaria y tecnología para la renovación técnica de los establecimientos industriales, o incluso las materias primas necesarias para la fabricación ${ }^{18}$.

Además, habría que añadir las circunstancias específicas que afectaban a la ya maltrecha industria corchera española al término de la guerra civil, que le habían hecho perder sus principales mercados frente a la industria lusa, que asumía por entonces el liderazgo en el negocio mundial tras más de ciento cincuenta años de dominio catalán ${ }^{19}$.

Gráfico 1: Exportaciones totales y de la rama corchera de España, 1929-1959 (en millones de pesetas constantes de 1913)

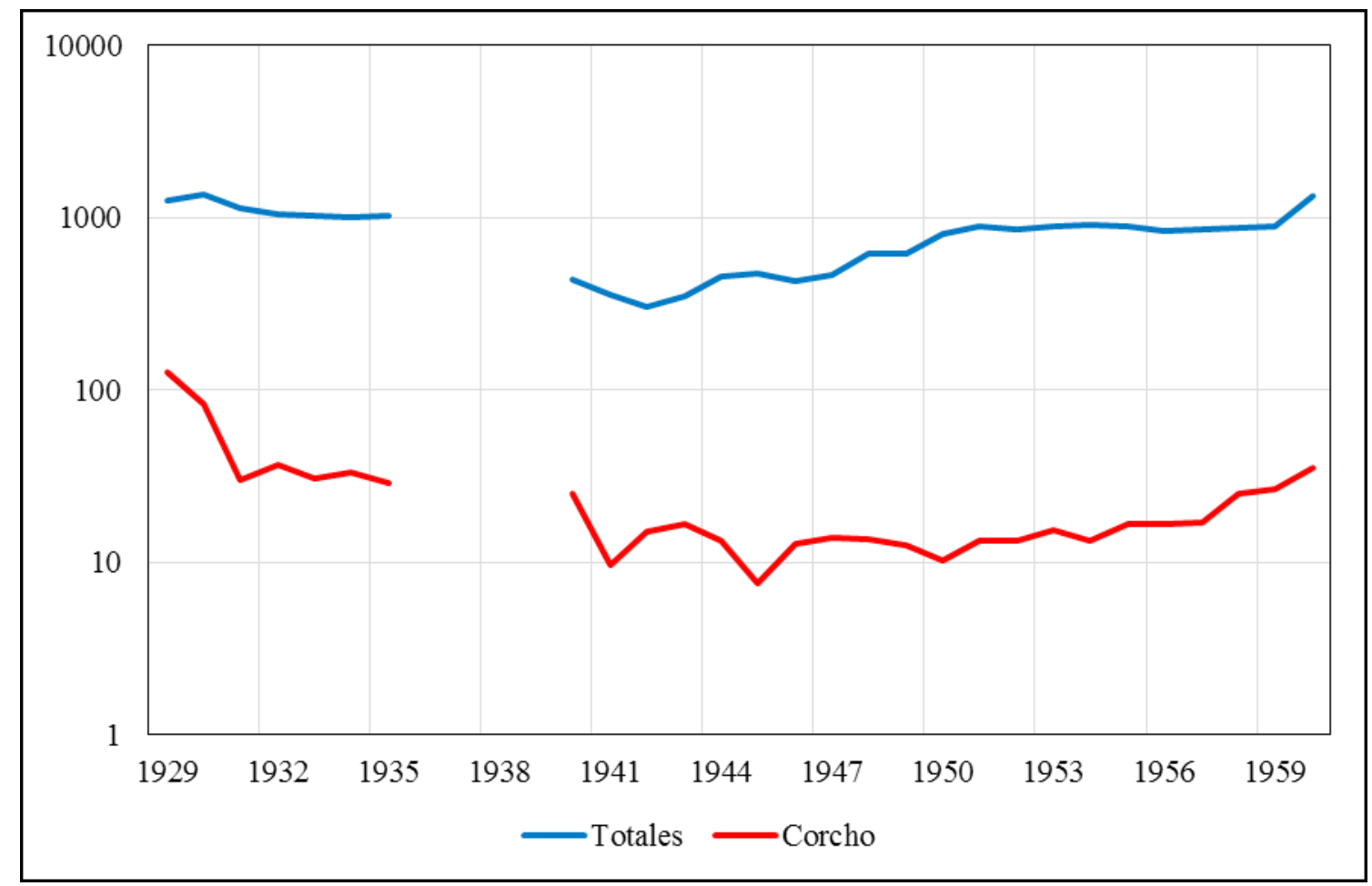

Fuente: para las exportaciones totales Tena, Antonio, "Sector exterior..." op. cit., pp. 604-605 (serie 1833 del cuadro 8.4); y para las corcheras Parejo Moruno, Francisco Manuel, El negocio de exportación... op. cit.

En este contexto es donde se deben situar las medidas de fomento a la exportación de productos corcheros que vamos a analizar. Que se aplicaran desde 1939 no quiere decir que

\footnotetext{
${ }^{18}$ Parejo Moruno, Francisco Manuel, El negocio de exportación... op. cit., pp. 244-251.

${ }^{19}$ Importantes mercados tradicionalmente españoles como Estados Unidos, Francia, Reino Unido, Argentina y Alemania pasaron entre 1929 y 1959 a ser abastecidos por Portugal, quedando la posición española muy debilitada; Parejo Moruno, Francisco Manuel, El negocio de exportación... op. cit., p. 163. Y la especialización española en el negocio, que siempre había estado en la producción y comercialización de manufacturas, se matizó sensiblemente al ganar el corcho bruto un mayor peso en la estructura de las exportaciones corcheras, justo lo contrario de lo ocurrido en Portugal; Branco, Amélia y Francisco Manuel Parejo Moruno, "Incentives or obstacles? The institutional aspects of the cork business in the Iberian Peninsula (1930-1975)", en Revista de Historia Económica-Journal of Iberian and Latin America Economic History, 1 (2008), p. 25.
} 
hasta entonces no se hubieran necesitado o solicitado. De hecho, las primeras voces solicitando medidas de apoyo a la exportación corchera son de comienzos del siglo xx y pertenecen a los industriales catalanes, que veían en estas medidas la única salida a la crisis de la taponería tradicional. Ilustres personajes del mundo corchero de la época como Primitivo Artigas se opusieron frontalmente:

\footnotetext{
"Algunos proponen como medio de remediar la crisis de la industria taponera conceder primas de exportación a los tapones, pero tal sistema debe, a nuestro juicio, emplearse en casos rarísimos y extremados, y tan sólo por corto tiempo, pues [...] serían muchas las industrias que, in continente, y con justicia [...] pedirían se les concediese parecido beneficio. Además, las primas sólo pueden establecerse en naciones ricas, por lo general, no en naciones pobres, como la nuestra" ${ }^{20}$.
}

A pesar de esta evidencia, en 1931 los corcheros españoles seguían jactándose de no haber tenido nunca que recurrir a los subsidios del Estado ${ }^{21}$. Pero, como afirmaba Ramiro Medir, la crisis de los años treinta hizo mella en el sector, y "aquella industria que siempre se había vanagloriado de no reclamar del Poder Público ayuda económica de ninguna clase [...] [tuvo que] modificar su actitud pidiendo primas a la exportación”22. La petición la formalizó el Fomento del Trabajo de la Industria Corcho-Taponera de Palafrugell (Gerona), y pretendía lograr del Estado un subsidio del 18 por 100 del valor de las exportaciones de tapones y demás corchos manufacturados. Es conveniente apuntar que la solicitud solo se realizaba para la venta al exterior de manufacturas y que el objetivo declarado de tales primas era cerrar la brecha existente entre los precios españoles y portugueses ocasionada por la desvalorización del Escudo. No obstante, la petición fue desestimada.

Tras la guerra civil el marco político e institucional cambió drásticamente, pero no los deseos de protección pública. La Ley de Protección a las Industrias de Interés Nacional de 24 de octubre de 1939 estableció la posibilidad de obtener beneficios fiscales para aquellos ramos industriales que hubieran sido declarados de interés para la nación. Desconocemos si la industria corchera recibió tal consideración, pero lo cierto es que los corcheros españoles ya se venían beneficiando de un régimen de "bonificaciones fiscales" (así eran denominadas oficialmente) desde unos meses antes. La medida la hizo pública la Comisión Arbitral del Corcho (CAC) — por entonces, el organismo público encargado de los asuntos corcheros- en abril de 1939, y sus efectos se extendían desde el 1 de enero anterior hasta el 1 de agosto del mismo año (cuadro 1). Oficialmente, el objetivo era devolver al exportador una parte de las cargas interiores soportadas, aunque en la práctica los incentivos representaban un subsidio directo a la exportación, pues consistían en devolver al agente un porcentaje del valor de las

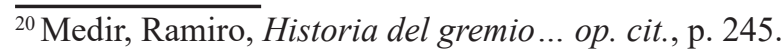

${ }^{21}$ Ibidem, p. 291.

${ }^{22}$ Ibídem, pp. 348-349.
} 
mercancías exportadas. Este régimen inicial de subsidios tenía la utilidad adicional para la administración de imprimir un estímulo mayor o menor al comercio de unos productos frente a otros con la mera modificación de los porcentajes de "bonificación”.

Cuadro 1: "Bonificaciones" a la exportación de productos corcheros (\% a aplicar sobre el valor FOB de la mercancía exportada)

\begin{tabular}{|l|c|c|c|c|c|c|}
\hline $\begin{array}{c}\text { Periodo de } \\
\text { vigencia }\end{array}$ & $\begin{array}{c}\text { Corchos de } \\
\text { trituración }\end{array}$ & $\begin{array}{c}\text { Corcho } \\
\text { aglomerado }\end{array}$ & $\begin{array}{c}\text { Manufacturas } \\
\text { de corcho } \\
\text { natural }\end{array}$ & $\begin{array}{c}\text { Cuadradillos, } \\
\text { láminas y } \\
\text { plantillas }\end{array}$ & $\begin{array}{c}\text { Corcho en } \\
\text { plancha } \\
\left(\mathbf{4}^{\mathbf{a}} \mathbf{y} \mathbf{5}^{\mathbf{a}}\right)\end{array}$ & $\begin{array}{c}\text { Corcho en } \\
\mathbf{p l a n c h a} \\
\left(\mathbf{1}^{\mathbf{a}} \mathbf{2} \mathbf{2}^{\mathbf{a}} \mathbf{y} \mathbf{3}^{\mathbf{a}}\right)\end{array}$ \\
\hline $\begin{array}{l}1-1-1939 / \\
1-8-1939\end{array}$ & 25 & 15 & 10 & 10 & 10 & 5 \\
\hline $\begin{array}{l}2-8-1939 / \\
9-8-1940\end{array}$ & $i ?$ & $i ?$ & $i ?$ & $i ?$ & $i ?$ & $i ?$ \\
\hline $\begin{array}{l}10-7-1940 / \\
10-10-1940\end{array}$ & 18 & 17 & $20(\mathrm{a})$ & 10 & 10 & 0 \\
\hline $\begin{array}{l}11-10-1940 / \\
31-7-1946\end{array}$ & $i ?$ & $i ?$ & $i ?$ & $i ?$ & $i ?$ & $i ?$ \\
\hline
\end{tabular}

Notas: (a) Tapones y discos.

Fuente: Archivo del Museu del Suro de Palafrugell [en adelante, AMSP], Sindicato Nacional de la Madera y del Corcho [en adelante, SNMC], Estadísticas e Informes, caja 13, "Circular n 28 de la Delegación Nordeste de la Rama Económica del Corcho".

Las razones que llevaron a la concesión de estos subsidios iniciales se exponen en un escrito de 18 de agosto de 1939 remitido por el presidente de la Rama Económica del Corcho (REC) - heredera de la CAC al frente de los asuntos corcheros- al Ministro de Industria y Comercio, en el cual se solicitaba una revisión al alza de los porcentajes de "bonificación"23. Por un lado, los subsidios a la exportación del corcho en plancha se justificaban por la necesidad de realizar el stock sobrante de 1937. Por otro lado, con el elevado porcentaje que se otorgó a los corchos de trituración (25 por 100) se trataba de compensar parcialmente las diferencias (en coste) existentes entre España y Portugal en las labores propias de la saca y en el transporte del corcho hasta las aduanas para su venta exterior. Y con razones similares se justificaba el 15 por 100 de subsidio aplicable a las exportaciones de aglomerado ${ }^{24}$.

Llama la atención que el porcentaje de subsidio más reducido - si se excluye el corcho en plancha de calidad - se otorgaba a la exportación de tapones y discos, a pesar de ser este uno de los ramos que estaba teniendo más problemas para subsistir en el mercado internacional. Según Salvador Robles Trueba (al frente de la REC), dado que los subsidios "fueron concedidos antes de liberarse Cataluña, se atendía [con ellos] sólo al desahogo de las industrias andaluzas” (y

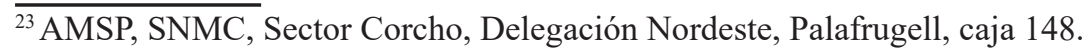

${ }^{24}$ Dentro de la industria transformadora del corcho se distinguen la del corcho natural y la del corcho aglomerado. La primera emplea el corcho en plancha preparado para fabricar tapones, discos y otras manufacturas de corcho natural. La segunda usa el corcho de mala calidad (el llamado refugo y el corcho bornizo, procedente del primer descorche) y los desperdicios de corcho para, una vez triturados, fabricar las manufacturas de aglomerado.
} 
extremeña) $)^{25}$, por lo que se puede concluir que, en este reparto inicial de "bonificaciones", la industria transformadora se vio perjudicada por el mero hecho de encontrarse concentrada en "territorio enemigo" (en Cataluña) hasta los instantes finales de la guerra. En suma, la administración franquista priorizó en este régimen inicial de subsidios los intereses de las regiones del suroeste, ligados a la producción forestal y a la industria preparadora, y por ende vinculados a la exportación del corcho sin manufacturar.

A raíz del referido escrito de 18 de agosto de 1939, se aprobaron nuevos porcentajes de subsidios para el trimestre entre el 10 de julio y el 10 de octubre de 1940. La novedad fue que las bonificaciones se restringieron solo a aquellas exportaciones realizadas en dólares USA, por lo que se trataba de una intervención estrictamente orientada a la captación de divisas. En otro orden, ignoramos qué ocurrió entre octubre de 1940 y julio de 1946, aunque intuimos que estas primeras intervenciones para el fomento de la exportación corchera fueron en general puntuales, a pesar de las reiteradas peticiones de los corcheros solicitando nuevas intervenciones ${ }^{26}$.

En definitiva, la situación de la industria corchera a mediados de la década de 1940 debió de ser crítica. A la vista del gráfico 1, no parece que las primeras medidas de fomento de las exportaciones corcheras hubieran sido efectivas. La provisionalidad de las mismas y, seguramente también, la insuficiencia de los porcentajes de subsidio aplicados impidió que las exportaciones del sector remontaran, de ahí que la dictadura tuviera que poner en funcionamiento un segundo bloque de medidas, esta vez más contundente y regular.

\section{La reactivación exportadora: el dumping corchero y las represalias norteamericanas (1946-1950)}

Una orden del Ministerio de Industria y Comercio de 31 de julio de 1946 instauró un nuevo régimen de subsidios a la exportación para diferentes productos corcheros (cuadro 2). Este régimen estuvo vigente desde la entrada en vigor de la orden ministerial (el 1 de agosto de 1946) hasta, al menos, marzo de 1950, y su finalidad era “aumentar la exportación, especialmente en manufacturas y materia prima de buena calidad, hasta un volumen de ventas totales de

\footnotetext{
${ }^{25}$ AMSP, SNMC, Sector Corcho, Delegación Nordeste, Palafrugell, caja 148.

${ }^{26}$ En un telegrama de 26 de mayo de 1946, remitido desde la oficina sindical corchera de Palafrugell (Gerona), se exponía con crudeza la urgencia de aplicar nuevos subsidios: "[...] ante la imposibilidad exportación debido precios elevados rogamos encarecidamente V. I. gestione presentación Consejo Ministros próximo viernes aprobación retornos punto caso contrario nos veremos obligados semana próxima visitar Gobernador Civil Gerona para solicitar cierre fábricas mayor parte industriales que no pueden sostener más esta situación”. AMSP, SNMC, Sector Corcho, Delegación Nordeste, Palafrugell, caja 148.
} 
130000000 de pesetas, como mínimo, en nueve meses" ${ }^{27}$. El subsidio debía ser concebido por los corcheros "como la diferencia entre costes y beneficios normales, por una parte, y el precio exterior, por otra. No [podía] consistir, por tanto, en un superprecio que se abon[ara] al exportador de artículos suficientemente valorizados en el exterior", como ocurría, por entonces, con los corchos de trituración y con el aglomerado para aislamiento ${ }^{28}$.

A semejanza de los incentivos aplicados en 1939 y 1940, el importe de los subsidios se calculaba aplicando el porcentaje correspondiente a los valores consignados en factura de las mercancías a exportar colocadas FOB en cualquier puerto español, en Lisboa o en la frontera hispano-francesa. Los porcentajes asignados debían ser revisados trimestralmente por una comisión presidida por el Director General de Comercio, la cual debía deliberar sobre la exportación de los diferentes productos del corcho y su situación en el mercado internacional, para, con criterio, fijar las nuevas primas a aplicar en el trimestre siguiente. Aunque, a la vista del cuadro 2, esta periodicidad en la revisión se incumplió sistemáticamente.

Obviando los penosos trámites burocráticos para solicitar y cobrar los subsidios ${ }^{29}$, es interesante analizar en qué medida sirvieron para mejorar la comercialización del corcho español, y si fomentaron el comercio de unos productos frente a otros, esto es si otorgaron mayor o menor protección a unos agentes frente a otros dentro de la cadena de valor de la industria. Sobre esto último, la orden de 31 de julio de 1946 otorgó los porcentajes más elevados a la exportación de tapones y discos de corcho natural, seguido del resto de manufacturas de corcho natural y de las de aglomerado — a excepción del aglomerado para aislamiento, que no se subsidió-.

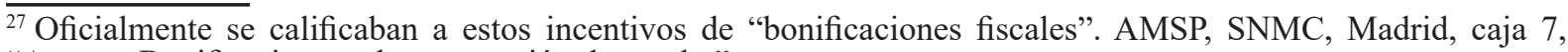
"Asunto: Bonificaciones a la exportación de corcho".

${ }^{28}$ Según el Ministerio de Industria y Comercio, salvo excepción autorizada, el precio exterior debía ser el precio vigente en el mercado internacional: "No inferior, porque ello representaría [...] [la] exportación de capitales en beneficio del comprador extranjero. Y tampoco superior, porque ello contraería las ventas". AMSP, SNMC, Madrid, caja 7, "Asunto: Bonificaciones a la exportación de corcho".

${ }^{29}$ Los detalles al respecto se describen en Parejo Moruno, Francisco Manuel, El negocio de exportación... op. cit., pp. 251-261.
} 
Cuadro 2: Subsidios a la exportación de productos corcheros, 1946-1950 (\% a aplicar sobre el valor FOB de la mercancía exportada)

\begin{tabular}{|c|c|c|c|c|c|c|}
\hline & $\begin{array}{l}1-8-1946 / \\
31-3-1947\end{array}$ & $\begin{array}{c}\text { 1-4-1947/ } \\
30-11-1947\end{array}$ & $\begin{array}{l}-12-1947 / \\
30-6-1948\end{array}$ & $\begin{array}{l}1-7-1948 / \\
14-5-1949\end{array}$ & $\begin{array}{l}15-5-1949 / \\
8-10-1949\end{array}$ & $\begin{array}{l}\text { 9-10-1949/ } \\
\text { Nov. } 1950\end{array}$ \\
\hline \multicolumn{7}{|l|}{ Corchos de trituración } \\
\hline - Refugo & 0 & 0 & 0 & 16 & 35 & 40 \\
\hline - Bornizo & 0 & 0 & 0 & 0 & 35 & 40 \\
\hline - Desperdicios & 0 & 0 & 0 & 16 & 35 & 60 \\
\hline \multicolumn{7}{|l|}{ Semimanufacturas } \\
\hline - Plancha $\left(\right.$ cal. $1^{\mathrm{a}}, 2^{\mathrm{a}}$ y $\left.3^{\mathrm{a}}\right)$ & $15(a)$ & 12,5 & 12,5 & 16 & 35 & 50 \\
\hline - Plancha (cal. $4^{a}$ y $5^{a}$ ) & $10(\mathrm{~b})$ & 12,5 & 12,5 & 16 & 35 & 50 \\
\hline - Cuadradillos $(>50 \mathrm{~mm})$ & 0 & 0 & 0 & 0 & 50 & 80 \\
\hline - Cuadradillos (<50 mm) & 24 & 24 & 24 & 25 & 50 & 80 \\
\hline - Serrín, granulados & 0 & 0 & 0 & 0 & 50 & 60 \\
\hline \multicolumn{7}{|l|}{ Manuf. corcho natural } \\
\hline - Tapones corrientes & 40 & 45 & 55 & 60 & 80 & 120 \\
\hline - Tapones champán & 28 & 28 & 40 & 50 & 80 & 120 \\
\hline - Discos & 40 & 45 & 55 & 60 & 80 & 120 \\
\hline - Flotadores & 40 & 45 & 55 & 55 & 80 & 120 \\
\hline - Láminas, plantillas & 24 & 24 & 40 & 40 & 70 & 100 \\
\hline - Papel de corcho & 28 & 28 & 55 & 55 & 80 & 120 \\
\hline - Otras especialidades (c) & $28(\mathrm{e})$ & $28(\mathrm{e})$ & $28(\mathrm{e})(\mathrm{d})$ & $30(\mathrm{e})$ & $50(f)$ & $80(\mathrm{~g})$ \\
\hline \multicolumn{7}{|l|}{ Manuf. corcho aglomerado } \\
\hline - Discos & 13 & 13 & 13 & 20 & 50 & 80 \\
\hline - Juntas & 13 & 13 & 13 & 20 & 50 & 80 \\
\hline - Aglomerado negro & 0 & 0 & 0 & 50 & 70 & 100 \\
\hline
\end{tabular}

Notas: (a) Corcho en plancha de buena calidad, de 5000 pesetas o más por tonelada; (b) Corcho en plancha de baja calidad, de entre 2500 y 4999 pesetas por tonelada; (c) Aplicable a las exportaciones de lana, corcho artístico, cajas, mangos, bolas, cartuchería, esterillas, carretes y demás especialidades no especificadas en los otros apartados; (d) A las losetas y zócalos de corcho se les asigna una prima del 24 por 100; (e) La exportación de baldosas de corcho recibía una prima del 30 por 100; (f) La exportación de baldosas, cascos tropicales y lana de corcho recibía una prima del 70 por 100; (g) La exportación de baldosas, cascos tropicales y lana de corcho recibía una prima del 100 por 100 .

Fuente: elaboración propia a partir de AMSP, SNMC, Sector Corcho, Delegación Nordeste, Palafrugell, caja 148, "Comercio de Corcho".

A priori, se aprecia un apoyo decidido a la industria transformadora por parte de las autoridades franquistas. Sin embargo, dicho apoyo no debió de ser pleno por dos motivos; primero, porque se otorgó un importante incentivo a la exportación de cuadradillos (de menos de $50 \mathrm{~mm}$ ), a partir de los cuales se obtenían algunas clases de tapones; y segundo, porque también se primó la exportación del corcho en plancha, incluso el de mejor calidad, lo que iba en detrimento de los intereses de la industria transformadora nacional. Sea como fuera, la exportación corchera reaccionó con los nuevos subsidios, pudiéndose reconquistar algunos de los mercados que habían sido arrebatados por la competencia portuguesa durante la guerra civili ${ }^{30}$.

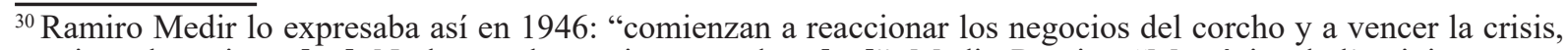
gracias a las primas [...]. No hay mal que cien años dure [...]". Medir, Ramiro, "Memòries de l'activitat surera (1939-1959)', en L'Estoig, 3 (1993), p. 51.
} 
La posición competitiva de la industria taponera española se vio reforzada con la entrada en vigor de nuevos porcentajes de subsidio el 1 de abril de 1947 (cuadro 2). En esta revisión apenas se vieron afectadas las exportaciones de las principales manufacturas de corcho natural, que vieron incrementadas sus respectivas primas en cinco puntos porcentuales. Las expectativas de la industria corchera española mejoraron hasta que, a finales de ese año, Estados Unidos —uno de los principales mercados de las manufacturas corcheras españolas, y el primero de nuestro corcho sin transformar- ${ }^{31}$ reprobó el régimen de subsidios español al considerarlo contrario a la libertad de mercado. La reprobación se concretó en el alza de los derechos arancelarios que pagaban los productos corcheros españoles a su entrada al país norteamericano.

El 18 de noviembre de 1947, Lorenzo Puértolas (Jefe del Sector Corcho del SNMC) expresaba: "las gestiones realizadas [...] al objeto de procurar la anulación del impuesto aduanero norteamericano no han alcanzado [...] ningún resultado positivo" ${ }_{32}$. Este nuevo arancel, según las autoridades norteamericanas, surgía de una previsión antidumping contenida en las leyes aduaneras estadounidenses. Sin embargo, la impresión de las autoridades comerciales españolas era diferente, y así lo denunciaba Puértolas: “[...] se trata de una medida cuya verdadera intención es más política que comercial y dimana del ambiente antiespañol que parece predominar en la Secretaría de Comercio Norteamericana", advirtiendo además "la indudable participación de Portugal en la medida restrictiva adoptada por los EEUU" ${ }^{33}$.

Y, probablemente, no le faltaba razón, pues los aranceles americanos se mantuvieron elevados incluso después de haber cesado el régimen de subsidios a la exportación corchera hacia este país. Al hilo de esto último, las represalias arancelarias de Estados Unidos sobre los corchos españoles habían llevado a la Asamblea Nacional Corchera a solicitar al Ministerio de Industria y Comercio, en noviembre de 1947, el cese definitivo de los subsidios de exportación hacia este mercado. Estas peticiones fueron atendidas por las autoridades españolas, que mediante una orden ministerial de 31 de enero de 1948 extinguieron el régimen de subsidios para las exportaciones realizadas a Estados Unidos ${ }^{34}$. Sin embargo, las medidas de represalia adoptadas por el gobierno estadounidense no cesaron de inmediato, por lo que los aranceles enfatizaron la desventaja competitiva española frente a la portuguesa en este mercado, la cual ya se había puesto de manifiesto con la mera supresión de los subsidios.

\footnotetext{
${ }^{31}$ Parejo Moruno, Francisco Manuel, El negocio de exportación... op. cit.

${ }^{32}$ AMSP, SNMC, Madrid, caja 7, "Comunicado de Lorenzo Puértolas, Jefe del SCSNMC sobre las gestiones realizadas al respecto del arancel decretado por Estados Unidos sobre los productos corcheros españoles".

${ }^{33}$ Ibidem.

${ }^{34}$ Parejo Moruno, Francisco Manuel, El negocio de exportación... op. cit., p. 469.
} 
Cuadro 3: Exportaciones corcheras españolas hacia EEUU (números índice con base 100 $=1947$ )

\begin{tabular}{|l|c|c|c|c|c|c|}
\hline & $\mathbf{1 9 4 7}$ & $\mathbf{1 9 4 8}$ & $\mathbf{1 9 4 9}$ & $\mathbf{1 9 5 0}$ & $\mathbf{1 9 5 1}$ & $\mathbf{1 9 5 2}$ \\
\hline Totales & $100(26)$ & $48(12)$ & $49(13)$ & $37(9)$ & $64(9)$ & $176(21)$ \\
\hline $\begin{array}{l}\text { - Mat. primas y } \\
\text { semimanufacturas }\end{array}$ & $100(50)$ & $65(23)$ & $64(21)$ & $51(18)$ & $69(18)$ & $217(47)$ \\
\hline $\begin{array}{c}\text { + Tablas y plancha } \\
\text { - Manufacturas }\end{array}$ & $100(45)$ & $58(18)$ & $65(18)$ & $47(14)$ & $78(17)$ & $180(40)$ \\
\hline + Tapones corcho natural & $100(16)$ & $25(5)$ & $30(6)$ & $18(3)$ & $57(5)$ & $121(9)$ \\
\hline
\end{tabular}

Notas: Entre paréntesis, peso relativo (en \%) de EEUU (como destino) en las exportaciones corcheras españolas (en las diferentes categorías consideradas en el cuadro).

Fuente: Parejo Moruno, Francisco Manuel, El negocio de exportación... op. cit.

Los efectos del conflicto diplomático con Estados Unidos son visibles en el cuadro 3. Las relaciones comerciales con Estados Unidos en lo que respecta al corcho se resintieron tanto que Juan Antonio Suanzes (Director del Instituto Nacional de Industria) se vio obligado a intervenir a finales de 1948 autorizando la realización de exportaciones corcheras hacia el país americano a través de la cuenta de compensación que con el mismo tenía abierta esta institución pública franquista 35 .

Al mismo tiempo, dada la importancia del mercado estadounidense la administración franquista contempló la revisión del régimen de subsidios, que permanecía intacto para el resto de destinos. La revisión prevista tenía una doble vertiente: primero, aumentar los incentivos a la exportación hacia el resto de mercados en el porcentaje suficiente como para compensar la pérdida del mercado norteamericano; y segundo, establecer un sistema de redistribución que permitiera abonar (indirectamente o de forma oculta) los subsidios a los exportadores hacia Estados Unidos. De esta forma, los exportadores corcheros, sin necesidad de recibir subsidios directamente, podrían seguir compitiendo en precio en el mercado norteamericano con los productos portugueses ${ }^{36}$.

Las intenciones anteriores concluyeron en una nueva revisión del régimen de subsidios que entró en vigor en diciembre de 1947, que apenas afectaba a los productos manufacturados, y del que ya no se beneficiaron — al menos no directamente — las exportaciones hacia Estados Unidos. Por tanto, parece que, a la par del objetivo de obtención de divisas, la dictadura sí optó por apoyar a la industria corchera en la comercialización de sus productos, al menos hasta junio de 1948, en que el régimen de primas a la exportación volvió a ser revisado.

Las posteriores revisiones del régimen de subsidios (entre julio de 1948 y noviembre de 1950) tienen distintas lecturas (cuadro 2). Primero, esconden la rotunda falta de competitividad

\footnotetext{
${ }^{35}$ Medir, Ramiro, "Memòries de l'activitat..." op. cit., p. 58.

${ }^{36}$ AMSP, SNMC, Madrid, caja 7, "Comunicado de Lorenzo Puértolas..." op. cit.
} 
del sector en los mercados exteriores. Segundo, constatan que el principal objetivo perseguido con los subsidios - el fomento exportador, o si se quiere, la protección de la industria corchera y sus exportaciones - a veces quedó supeditado a la consecución de otros fines como la captación masiva de divisas. Lo atestiguan dos circunstancias; una, el mayor crecimiento relativo de los subsidios aplicados a la venta del corcho en plancha; y otra, los fuertes incentivos aplicados a la exportación de los corchos de trituración —-materia prima de la industria de aglomerados-, que hasta junio de 1948 no habían recibido prima alguna ${ }^{37}$.

Una prueba al respecto se encuentra en la oposición del Instituto Español de Moneda Extranjera a realizar el pago de la prima a aquellas exportaciones efectuadas en pesetas, es decir, no generadoras de divisas ${ }^{38}$. Parece evidente, entonces, que el objetivo de proteger la industria se vio interceptado en ocasiones por las urgencias en materia de divisas que vivió la administración franquista, además de por la discrecionalidad resultante de las presiones ejercidas desde los lobbies forestales y preparadores, deseosos de exportar sus corchos sin manufacturar.

En noviembre de 1950, el régimen de subsidios a la exportación de productos corcheros fue suprimido. En compensación, estos productos se incorporaron a un mecanismo de cambios múltiples que la dictadura había puesto en funcionamiento en 1948, mediante el cual se reservaban porcentajes de divisa para su negociación en bolsa en las diferentes operaciones comerciales.

\section{El corcho en el mecanismo de cambios múltiples (1950-1957)}

La mayoría de los trabajos que han estudiado la economía española en las décadas de 1940 y 1950 coinciden en señalar a la política cambiaria como el principal motivo del estancamiento exportador ${ }^{39}$. El argumento adquiere sentido desde 1941, año en que las autoridades franquistas optaron por fijar un cambio oficial de 10,95 pesetas/dólar USA, que se mantuvo firme hasta

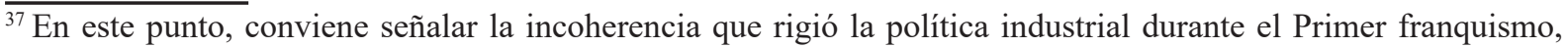
cuyo rumbo incierto estuvo condicionado, y en gran medida definido, por el triunfo de las presiones ejercidas por los diversos grupos de presión existentes. Véase al respecto Pires Jiménez, Luis Eduardo, "La libertad económica en el primer franquismo: el control de la actividad empresarial y la política industrial”, en La Albolafia: Revista de Humanidades y Cultura, 1 (2014), pp.69-96.

${ }^{38}$ Parejo Moruno, Francisco Manuel, El negocio de exportación ... op. cit., pp. 469-470.

${ }^{39}$ Asensio, María Jesús, El proceso de apertura exterior de los cincuenta y el arancel de 1960, tesis doctoral, Universidad de Zaragoza, 1995; Serrano, José María y María Jesús Asensio, "El ingenierismo cambiario..." op. cit.; Martínez Ruiz, Elena, El control de cambios... op. cit.
} 
1948, año en que comenzó a funcionar en España un sistema de cambios múltiples ${ }^{40}$. Ajena a la inflación que sufrió la economía española en los años cuarenta, la dictadura optó por mantener inalterable el valor de la peseta, asociando su fortaleza a la ganancia de prestigio internacional ${ }^{41}$. El balance final fue una pérdida de competitividad de los productos españoles, a pesar de los estímulos que se concedieron a algunos sectores exportadores, analizados en los apartados anteriores.

La sobrevaloración oficial de la peseta es apreciable si se compara con su cotización libre en el mercado internacional ${ }^{42}$. A la vista del gráfico 2 , es evidente que el cambio oficial se mantuvo inalterable de forma artificial, sin recoger la fuerte depreciación de la moneda española en el mercado internacional desde 1943. Este sostenimiento a ultranza del tipo de cambio contrasta con lo ocurrido en otros países europeos, que tras la guerra mundial optaron por devaluar sus monedas para recuperar los niveles de exportación previos al conflicto.

El sistema de cambios múltiples instaurado en diciembre de 1948 consistió en la aplicación de cambios diferenciales a las operaciones de exportación e importación de diferentes productos. El cambio oficial siguió vigente en aquellas operaciones a las que no se asignó ningún cambio especial. Por término medio, los cambios especiales aplicados de inicio supusieron una devaluación de la peseta del 36 por 100, aunque aquellos se fueron revisando continuamente a lo largo de la década de 1950, hasta el restablecimiento del cambio único a finales de la misma ${ }^{43}$. Otro incentivo para el exportador radicaba en los porcentajes de divisas que se le permitía negociar en bolsa, que, en cierto modo, acababa siendo una mejora adicional del cambio especial asignado.

\footnotetext{
${ }^{40}$ Realmente, el cambio oficial de 10,95 pesetas/dólar USA se mantuvo hasta 1957, si bien a finales de la década de 1940 hubo una tibia relajación cambiaria que permitió una cierta devaluación encubierta de la peseta, tal y como se explica en Serrano, José María y María Jesús Asensio, "El ingenierismo cambiario...” op. cit.

${ }^{41}$ Catalan, Jordi, "Reconstrucción política económica..." op. cit., p. 368.

${ }^{42}$ Es cierto, como se verá a continuación, que la mayor parte de los productos corcheros estuvieron sometidos en sus operaciones de exportación a un tipo de cambio efectivo (real) más remunerador que el oficial de las 10,95 pesetas por dólar USA, aunque lejano de la cotización libre de la peseta en el mercado internacional.

${ }^{43}$ Serrano, José María y María Jesús Asensio, "El ingenierismo cambiario..." op. cit.
} 
Gráfico 2: Tipo de cambio oficial de la peseta con el dólar USA y cotización libre de la peseta en el mercado de Tánger (peseta/dólar USA)

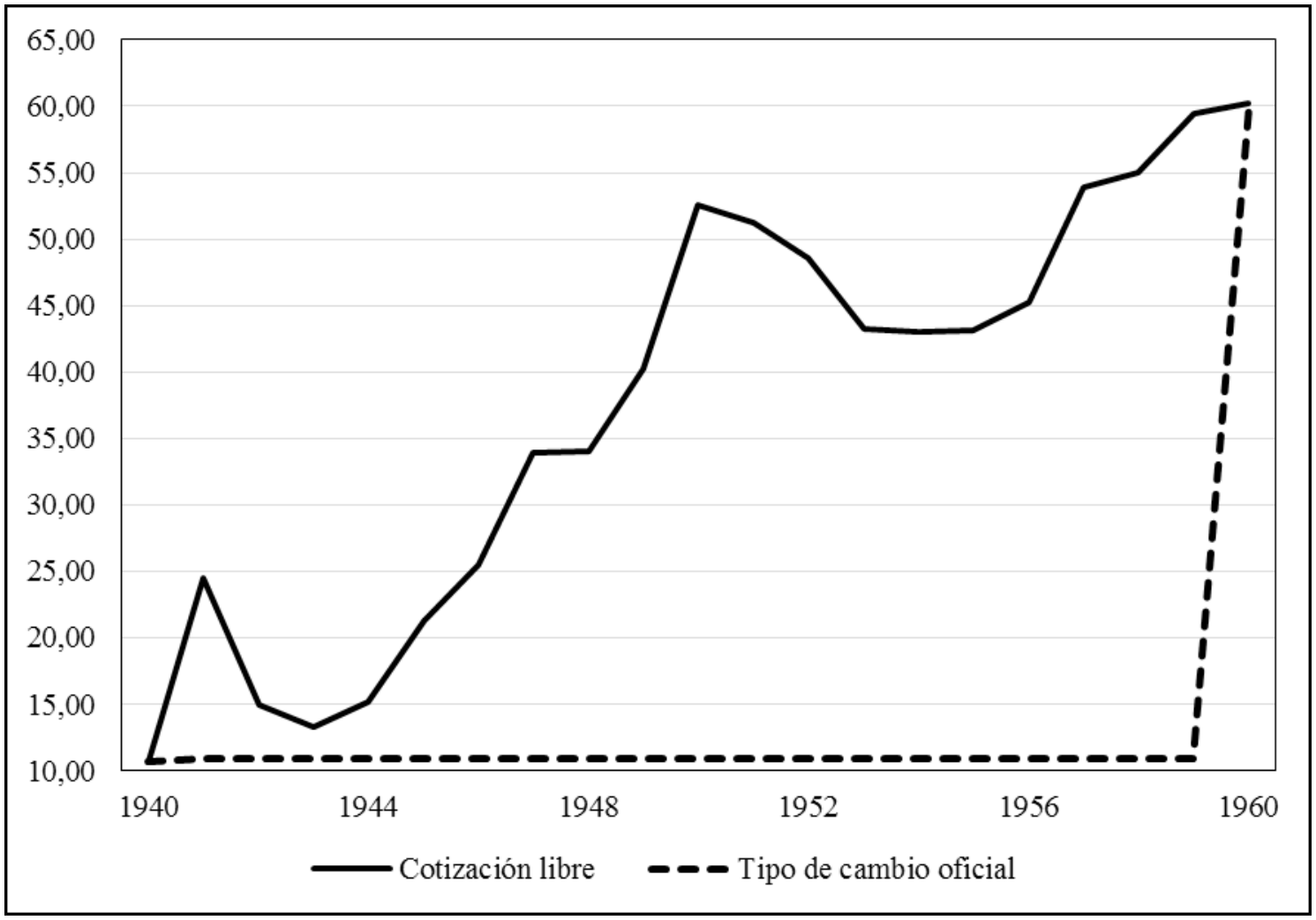

Fuente: Martín Aceña, Pablo y María de los Ángeles Pons, "Sistema monetario y financiero", en Carreras, Albert y Xavier Tafunell (coords.), Estadísticas históricas de España, siglos XIX-XX. Vol. II, Pamplona, Fundación BBVA, 2005, pp. 703-706.

La ejecución de este complejo instrumental cambiario se realizó estableciendo diversos grupos de mercancías de exportación e importación, en un número que fue modificándose con el tiempo ${ }^{44}$. A cada uno de ellos la administración le asignó un cambio especial determinado -y el porcentaje de divisas negociable correspondiente-, más o menos remunerador según el interés de aquella en fomentar su exportación o importación. Los productos corcheros no fueron incluidos inicialmente en ninguno de los grupos de importación, pero sí en los de exportación, entre los que las distintas calidades del corcho se distribuyeron según su grado de transformación.

En el cuadro 4 se presentan los tipos de cambio especiales y los porcentajes negociables de divisa que estuvieron vigentes para los productos corcheros durante el período de cambios múltiples. En primer lugar, debe advertirse que por tipo de cambio efectivo entendemos aquel que resultaba de aplicación real al exportador, teniendo en cuenta el cambio especial asignado

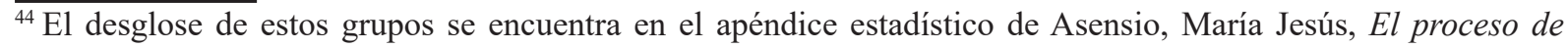
apertura exterior... op. cit.
} 
y el porcentaje de negociación en bolsa autorizado ${ }^{45}$. Salta a la vista que los tipos de cambios iniciales fueron modificados en varias ocasiones hasta 1959, bien porque las autoridades decidieron abaratar la peseta en algunos de los grupos establecidos o porque las mercancías corcheras saltaron a grupos con un mejor tratamiento cambiario. Tanto en los cambios establecidos como en los porcentajes de reserva de divisa para negociación en bolsa se pone de manifiesto el intento de la dictadura por fomentar la exportación de los productos con mayor grado de transformación ${ }^{46}$, al menos inicialmente.

Las modificaciones introducidas en noviembre de 1951 no fueron relevantes, pero sí las que tuvieron lugar en julio de 1953. En ese momento, las autoridades comerciales españolas metieron en el mismo grupo (Grupo 3 de las exportaciones) a las manufacturas y semimanufacturas corcheras de todas clases, por lo que el grueso de la industria española (preparadora y transformadora) quedó sometida al cambio especial de 21,90 pesetas/dólar USA y a la reserva del 50 por 100 de las divisas. Desde febrero de 1957, las definiciones de los productos del corcho que aparecen en los grupos de exportación son muy genéricas, por lo que no es posible precisar, por ejemplo, si el corcho en plancha (preparado) o el granulado de corcho tuvieron el tratamiento de materia prima o de manufactura. Ambos capítulos, no obstante, mejoraron su tratamiento cambiario de cara a la exportación. Hasta que en abril de 1957 se puso fin al mecanismo de cambios múltiples, instaurándose un tipo de cambio único de 42 pesetas/dólar USA, todavía alejado de las 54 pesetas/dólar USA a que cotizaba la moneda española en el mercado de Tánger.

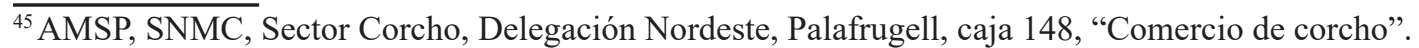

${ }^{46}$ Los cambios efectivos más altos son los relativos a la exportación de tapones y discos, y aunque a casi todos los productos corcheros se asignó una peseta más devaluada que la del cambio oficial, el trato más favorable fue para los productos industriales. Además, la materia prima (corcho sin preparar) fue excluida inicialmente de estos incentivos cambiarios.
} 
Cuadro 4: Tipos de cambio aplicables a las exportaciones de productos corcheros y porcentajes de divisas autorizados para su negociación en bolsa (peseta/dólar USA)

\begin{tabular}{|c|c|c|c|c|c|}
\hline & Mercancías objeto de comercio & Te X & $\%$ Divisa & $\begin{array}{c}\text { Tc X } \\
\text { (efectivo) }\end{array}$ & Tc libre \\
\hline $\begin{array}{l}\text { De } 1941 \text { a } \\
6-11-1950\end{array}$ & — Todas las calidades & 10,95 & 0 & 10,95 & $24-52$ \\
\hline \multirow{5}{*}{$\begin{array}{l}\text { De 6-11-1950 } \\
\text { a } 31-10-1951\end{array}$} & - Corcho en plancha & 16,43 & 10 & 18,77 & \multirow{5}{*}{51} \\
\hline & $\begin{array}{c}\text { - Manufacturas y semimanufacturas } \\
\text { no comprendidas en otros apartados } \\
\text { (cuadradillos) }\end{array}$ & 21,90 & 10 & 23,67 & \\
\hline & $\begin{array}{c}\text { - Aglomerado negro, blanco y sus } \\
\text { manufacturas }\end{array}$ & 21,90 & 30 & 27,28 & \\
\hline & - Corcho en papel (bobinas) & 21,90 & 40 & 29,08 & \\
\hline & $\begin{array}{l}\text { - Tapones, discos de corcho natural y } \\
\text { flotadores }\end{array}$ & 21,90 & 50 & 30,42 & \\
\hline \multirow{5}{*}{$\begin{array}{l}\text { De } 1-11-1951 \\
\text { a } 1-7-1953\end{array}$} & - Materia prima & 21,90 & 0 & 21,90 & \multirow{5}{*}{51} \\
\hline & - Corcho semimanufacturado & 21,90 & 10 & 23,67 & \\
\hline & $\begin{array}{l}\text { - Manufacturas salvo papel en bobinas, } \\
\text { tapones, discos, flotadores y especialidades }\end{array}$ & 21,90 & 30 & 27,28 & \\
\hline & $\begin{array}{c}\text { - Papel en bobinas, tapones, discos y } \\
\text { flotadores }\end{array}$ & 21,90 & 50 & 30,42 & \\
\hline & — Especialidades (artículos de artesanía) & 21,90 & 70 & 33,84 & \\
\hline \multirow{3}{*}{$\begin{array}{l}\text { De } 1-7-1953 \\
\text { a } 17-2-1957\end{array}$} & — Materia prima y desperdicios & 21,90 & 0 & 21,90 & \multirow{3}{*}{45} \\
\hline & $\begin{array}{l}\text { - Corcho manufacturado y } \\
\text { semimanufacturado de todas clases }\end{array}$ & 21,90 & 50 & 30,40 & \\
\hline & — Corcho de artesanía & 21,90 & 70 & 33,80 & \\
\hline \multirow{2}{*}{$\begin{array}{l}\text { De } 18-2-1957 \\
\text { a } 12-4-1957\end{array}$} & - Materia prima & 21,90 & 30 & 27,01 & \multirow{2}{*}{54} \\
\hline & - Corcho manufacturado & 21,90 & 90 & 37,24 & \\
\hline \multirow{2}{*}{$\begin{array}{l}\text { De } 13-4-1957 \\
\text { a } 31-12-1957\end{array}$} & - Corcho en bruto & 42 & 0 & (a) 31 & \multirow{2}{*}{54} \\
\hline & - Corcho manufacturado & 42 & 0 & 42 & \\
\hline \multirow{2}{*}{$\begin{array}{l}\text { De } 1-1-1958 \\
\text { a } 14-2-1959\end{array}$} & - Corcho en bruto & 42 & 0 & (b) 36 & \multirow[b]{2}{*}{58} \\
\hline & $\begin{array}{l}\text { - Manufacturas y semimanufacturas de } \\
\text { corcho }\end{array}$ & 42 & 0 & (c) 45 & \\
\hline \multirow{2}{*}{$\begin{array}{l}\text { De } 15-2-1959 \\
\text { a } 20-7-1959\end{array}$} & - Materia prima & 42 & 0 & (b) 36 & \multirow{2}{*}{59} \\
\hline & - Manufacturas y desperdicios & 42 & 0 & (d) 50 & \\
\hline 20-7-1959 & Todas las calidades (cambio único) & 60 & 0 & 60 & 59 \\
\hline
\end{tabular}

Abreviaturas: Tc X =Tipo de cambio especial peseta/dólar USA establecido para las operaciones de exportación; \% Divisa $=$ Porcentaje de divisas de la operación que la administración autorizaba a negociar en bolsa; Tc X (efectivo) =Tipo de cambio efectivo para el exportador; Tc libre =Cotización aproximada de la peseta respecto al dólar USA en el mercado libre de Tánger.

Notas: (a) Cambio de 42 pesetas/dólar USA, menos un retorno de 11 pesetas/dólar USA; (b) Cambio de 42 pesetas/ dólar USA, menos un retorno de 6 pesetas/dólar USA; (c) Cambio de 42 pesetas/dólar USA, más una prima de 3 pesetas/dólar USA; (d) Cambio de 42 pesetas/dólar USA, más una prima de 8 pesetas/dólar USA.

Fuente: AMSP, SNMC, Sector corcho. Delegación Nordeste, Palafrugell, caja 148, "Comercio de corcho"; y Martín Aceña, Pablo y María de los Ángeles Pons, "Sistema monetario y..." op. cit., pp. 703-706.

Desde el SNMC se habían ya manifestado en contra de la política de cambios múltiples, manifestando su deseo de volver al cambio único y real, apelando a 
"la insuficiencia de los cambios aplicados a las exportaciones corcheras en relación con la cotización internacional de la peseta, [que] había provocado una profusión de cuentas combinadas, compensaciones, especulaciones, fraudes y falsificaciones, [...] que nada tenía que ver con la honrada industria tradicional" ${ }^{47}$.

También apuntaban que el sistema de cambios especiales había mostrado "durante el largo periodo de su vigencia, no ya una total inoperancia, sino, incluso, unos resultados desastrosos, completamente contrarios a los fines que se pretendieron lograr con su implantación"48.

Y no les faltaba razón. Basta para entenderlo la mera comparación de las dos últimas columnas del cuadro 4. Es justo reconocer que el mecanismo de cambios múltiples pudo suponer un empujón para una industria exportadora como la corchera, pues abarató la peseta con creces respecto al cambio oficial. Sin embargo, cualquier industria orientada al exterior perdió con la intervención, pues la cotización libre de la peseta en el mercado internacional siempre estuvo muy por encima del cambio oficial, del cambio especial, e incluso del cambio efectivo o real de la operación. Además, al mejorarse también las condiciones cambiarias para los exportadores de corcho sin manufacturar se puede entender que el objetivo prioritario de proteger a la industria nacional transformadora no siempre fue tal, pues quedó sometido, de nuevo, a otros, como la generación de divisas o como la defensa de la exportación del corcho sin transformar, demandada por los agentes forestales y preparadores del negocio. En definitiva, parece que la salud del país en materia de divisas, por un lado, o la discrecionalidad en favorecer a unos u otros grupos de presión, por el otro, evitaron la consecución del objetivo estructural de proteger la fabricación, en una industria, por otra parte, muy venida a menos.

\section{Conclusiones}

Durante el período 1929-1959 la industria corchera española perdió el liderazgo en el negocio corchero mundial, que había ocupado casi desde su origen. Este liderazgo se había cimentado en la exportación, habida cuenta de la debilidad de la demanda interna, de modo que las exportaciones corcheras españolas crecieron de forma ininterrumpida hasta alcanzar en la década de 1920 sus mayores registros históricos, convirtiéndose el corchero en uno de los principales capítulos de la exportación tradicional en España. La gran depresión de 1929, primero, la guerra civil, más tarde, y el intervencionismo autárquico a que se enfrentó el sector

\footnotetext{
$\overline{47}$ AMSP, SNMC, Estadísticas e Informes, caja 352, "Informe de la Junta Económica Provincial del Sindicato de Madera y Corcho (Grupo Corcho)".

${ }^{48}$ Ibídem.
} 
desde 1939, hicieron sucumbir la exportación corchera, perdiendo la industria española su posición hegemónica de manos de la competencia portuguesa.

Todo ello ocurrió en un periodo que no estuvo exento de medidas públicas para el fomento de la exportación corchera. De hecho, nada más terminar la guerra civil, las autoridades franquistas subsidiaron la venta al exterior de determinados productos corcheros, si bien de forma poco eficaz, inicialmente, tal vez por la discontinuidad de los subsidios y por su insuficiencia. Ello explica que la serie de exportaciones corcheras españolas mantuviera su tendencia decreciente durante los años en que estuvieron vigentes, y también que los industriales corcheros se pasaran la primera mitad de la década de 1940 solicitando nuevas y mayores medidas de apoyo.

Estas llegaron en 1946 a través de un nuevo régimen de subsidios, del cual se beneficiaron los exportadores corcheros hasta 1950. Lo significativo de este nuevo régimen es que constituyó un dumping en toda regla. La cuantía de los subsidios aplicados, su revisión periódica (siempre al alza) y su regularidad denotan la apuesta decidida de la administración franquista por el fomento exportador. Aunque, en verdad, su efectividad solo puede ser calificada de modesta, dada la evolución de la serie de exportación corchera después de 1946, y dados también los problemas comerciales que generó con Estados Unidos, que no dudó en aplicar medidas antidumping contra los corchos españoles para contrarrestar el régimen español de subsidios.

En otro orden, el objetivo institucional de proteger a la industria nacional —esgrimido por las autoridades al establecerse el régimen de subsidios de 1946- pasó en ocasiones a un segundo lugar por la mala situación del Tesoro español, o por la preeminencia de los intereses de otros agentes (forestales y preparadores), orientados hacia la exportación del corcho sin transformar. En este sentido, aunque es cierto que los industriales recibieron cuantiosos incentivos para exportar sus manufacturas, no lo es menos que también se primó de forma considerable la exportación del corcho sin transformar de calidad, teniendo esto dos implicaciones negativas para la industria: primero, la menor oferta de corcho y su consiguiente encarecimiento en el mercado nacional; y segundo, el mejor aprovisionamiento de materia prima de calidad de las industrias competidoras extranjeras, muchas de las cuales no tenían corcho autóctono para trabajar y debían importarlo.

Después, con la liberalización económica internacional debió de resultar anacrónico mantener un régimen de subsidios directos a la exportación, como el iniciado en 1946. De esta forma, en 1950 se puso fin a esta intervención, incorporándose los productos corcheros al mecanismo de cambios múltiples, vigente (para otros muchos productos) desde 1948. Con ello se pasó a un régimen de subvenciones encubiertas basado en dos premisas: la primera, la mejora sustancial del tipo de cambio aplicable a la exportación de los diferentes productos corcheros; y la segunda, la posibilidad otorgada al exportador de negociar en bolsa un porcentaje de las divisas obtenidas con la exportación. Cambió el instrumento para el fomento exportador, pero no la filosofía. De hecho, aunque los cambios establecidos para las manufacturas corcheras tiraron 
al alza de su exportación, los asignados a la materia prima y al corcho semielaborado también constituyeron un incentivo notable para su envío al exterior. De esta forma, los incentivos que recibió la exportación de manufacturas pudieron haberse visto neutralizados por la escasez de corcho para trabajar en el mercado nacional, acuciante en algunos años de las décadas de 1940 y 1950 .

Por último, el hecho de que los incentivos a la exportación llegaran a alcanzar enormes proporciones conduce a dos reflexiones. La primera de ellas alude a la enorme desventaja competitiva que debieron de tener los productos corcheros españoles en el mercado mundial durante la autarquía franquista. El hecho de que se establecieran porcentajes de subsidio de hasta el 120 por 100 del valor exportado para las manufacturas de corcho natural es indicativo de ello. Probablemente, el mantenimiento artificial del tipo de cambio oficial durante la década de 1940 fue letal para las exportaciones corcheras, las cuales solo pudieron remontar tímidamente cuando pudieron beneficiarse del dumping corchero del período 1946-1950. Esta fijación en mantener una peseta sobrevalorada fue el clavo ardiendo al que se agarró la dictadura para casar la demanda y la oferta de divisas, aspecto sobre el que versa la segunda reflexión. Y es que la delicada situación de divisas que atravesó España hizo que las medidas del fomento exportador aplicadas sobre el corcho no fueran siempre las que la industria transformadora habría deseado. La urgencia de allegar divisas, sin discernir si estas procedían del corcho manufacturado o sin manufacturar, fue una constante, junto a la negativa a conceder subsidios a las exportaciones no generadoras de estas. Así, el objetivo sectorial del fomento de las exportaciones corcheras quedó, por momentos, supeditado a un objetivo más urgente, el de generar divisas, no pudiéndose evitar el fracaso de la fabricación del corcho en España. 